\title{
Peroxiredoxins in Regulation of MAPK Signalling Pathways; Sensors and Barriers to Signal Transduction
}

\author{
Heather R. Latimer, and Elizabeth A. Veal*
}

\begin{abstract}
Peroxiredoxins are highly conserved and abundant peroxidases. Although the thioredoxin peroxidase activity of peroxiredoxin (Prx) is important to maintain low levels of endogenous hydrogen peroxide, Prx have also been shown to promote hydrogen peroxide-mediated signalling. Mitogen activated protein kinase (MAPK) signalling pathways mediate cellular responses to a variety of stimuli, including reactive oxygen species (ROS). Here we review the evidence that Prx can act as both sensors and barriers to the activation of MAPK and discuss the underlying mechanisms involved, focusing in particular on the relationship with thioredoxin.
\end{abstract}

\section{INTRODUCTION}

Reactive oxygen species (ROS) are damaging, highly reactive, reduced forms of oxygen, produced in a wide range of physiological processes, including as a by-product of aerobic respiration in the mitochondria, and following exposure of cells to environmental agents such as UV light and drugs/xenobiotics (Gutteridge and Halliwell, 1999). Over recent years, rather than simply acting as toxic agents, it has been established that lessreactive $\mathrm{ROS}$, particularly $\mathrm{H}_{2} \mathrm{O}_{2}$, also play positive roles as signalling molecules. Key to ROS-signalling functions is the ability to selectively regulate the activity of specific proteins, for example, by oxidising deprotonated cysteine residues, such as are found in the catalytic sites of many enzymes. This selective reactivity is one of the features making $\mathrm{H}_{2} \mathrm{O}_{2}$ well-suited as a signalling molecule (Winterbourn, 2008). Indeed, $\mathrm{H}_{2} \mathrm{O}_{2}$ signals have been shown to promote diverse biological responses, from stomatal opening in plants to cell migration in wound healing [For reviews see (Veal and Day, 2011)] and (Holmstrom and Finkel, 2014). However, the most fundamental and universal response to increases in ROS is to limit organismal damage by

Institute for Cell and Molecular Biosciences, Newcastle University, Framlington Place, Newcastle upon Tyne, NE2 4HH, UK

${ }^{\star}$ Correspondence: e.a.veal@ncl.ac.uk

Received 30 November, 2015; accepted 3 December, 2015; published online 25 January, 2016

Keywords: kinase, peroxiredoxin, phosphatase, redox, thioredoxin increasing the levels of detoxification and repair enzymes or initiating apoptosis. Many of these protective responses are mediated by MAPK pathways (Fig. 1). As we discuss below, redox-active cysteines have been shown to play an important part in the activation of these pathways.

Cells have evolved a variety of enzymes which remove ROS before they are able to cause oxidative damage (Bokov et al., 2004; Gutteridge and Halliwell, 1999). Redox-active cysteines are involved in the activity of many of these enzymes. For example, peroxiredoxins (Prx) are a family of extremely abundant, thioredoxin peroxidase enzymes. Prx are themselves highly reactive with $\mathrm{H}_{2} \mathrm{O}_{2}$ using the reversible oxidation of cysteine residues to reduce peroxides, including $\mathrm{H}_{2} \mathrm{O}_{2}$. During the cataIytic mechanism of typical 2-Cys $\mathrm{Prx}$, the peroxidatic cysteine becomes oxidised to a sulphenic acid, which then reacts with the resolving cysteine of a neighbouring Prx molecule, forming an intermolecular disulphide bond. The resulting Prx disulphides are reduced by thioredoxin using electrons from NADPH (Fig. 2A) [For a review see (Wood et al., 2003)]. Thioredoxin family proteins are oxidoreductases with broad substrate specificity, acting as cofactors in the activity of many other enzymes and participating in the reduction of many transcription factors and signalling molecules (Holmgren and Lu, 2010). However, the abundance of Prx and the high affinity of thioredoxin for Prx disulphides can render Prx disulphides the prevalent thioredoxin substrate when $\mathrm{H}_{2} \mathrm{O}_{2}$ levels increase (Day et al., 2012). Hence, the activities of $\mathrm{Prx}$ and thioredoxin in $\mathrm{H}_{2} \mathrm{O}_{2}$ signalling are inextricably linked. Here we will discuss the different roles that have been identified for Prx and thioredoxin in the regulation of mitogen activated protein kinase (MAPK) pathways. In some cases, Prx and thioredoxin have been shown to act as barriers to signal transduction, whereas in others Prx and thioredoxin are required for effective signalling. We will discuss the underlying mechanisms that give rise to these apparent contradictions.

\section{MAPK PATHWAYS}

In eukaryotes, conserved MAPK pathways mediate responses to various stimuli. In unicellular eukaryotes these MAPK pathways promote changes in gene expression that are vital for adaptation and growth under changing environmental conditions (Toone and Jones, 1998). MAPK signalling pathways are also important mediators of growth factors and 
Mammals:

Generic MAPK pathway :

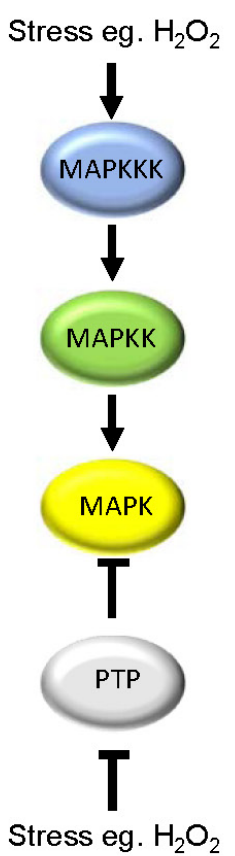

Oxidative and osmotic stress

Cytokines e.g.TNFa UV irradiation

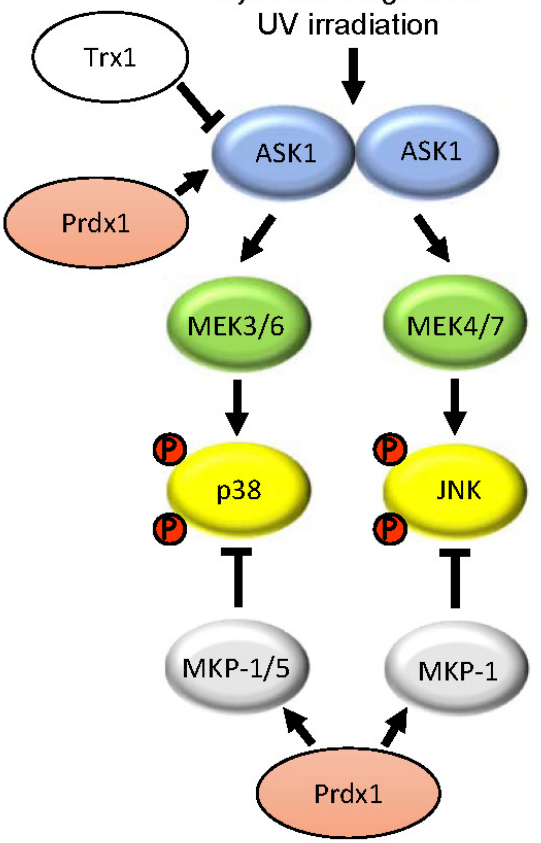

Fission yeast (Schizosaccharomyces pombe):

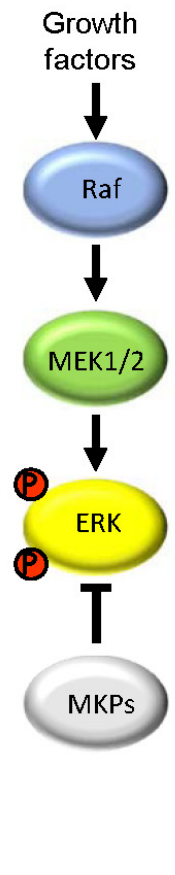

Oxidative and osmotic stress, UV irradiation

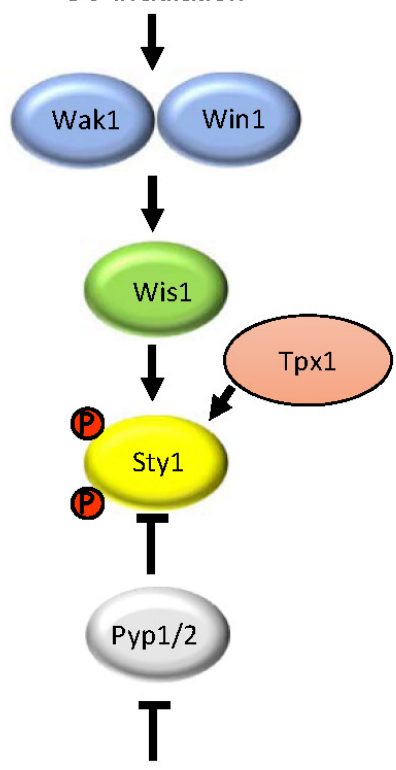

Heat stress

Fig. 1. Mitogen activated protein kinase (MAPK) signalling pathways mediate responses to a variety of stimuli, including ROS. MAPK pathways consist of a series of kinases: MAPKKK, MAPKK and MAPK, which are sequentially activated by phosphorylation. Three groups of MAPK, p38, JNK (c-Jun N-terminal kinase) and ERK (extracellular signal-regulated kinase), are preferentially activated by different MAPKK and MAPKKK. The activity of each kinase is also controlled by phosphatases. For example, to attenuate MAPK activity, protein tyrosine phosphatases (PTPs) dephosphorylate MAPK. Oligomerisation activates ASK1 by promoting autophosphorylation. Inactivation of phosphatases is an alternative mechanism to increase MAPK activity. For example, heat stress activation of the Sty1(Spc1) pathway in the fission yeast Schizosaccharomyces pombe involves inactivation of the PTP Pyp1 (Nguyen and Shiozaki, 1999). Steps in pathways which have been shown to be regulated by peroxiredoxins (pink) and/or thioredoxin (Trx1) are also indicated.

cytokine responses. For example, three MAPKs, JNK, p38 and ERK, all become activated following exposure to the cytokine, tumour necrosis factor- $\alpha$ (TNF- $\alpha$ ). MAPK pathways consists of a series of protein kinases, which become sequentially activated by phosphorylation (Fig. 1) [For a review see (Marshall, 1994)]. Although some of the protein targets mediating the effects of MAPK remain unclear, MAPK have been found to phosphorylate a variety of target proteins, including transcription factors [For a review see (Sabio and Davis, 2014)]. Work to understand the activation of MAPK has largely focussed on how the phosphorylation of upstream kinases is triggered. However, there is some evidence that the regulation of dephosphorylation reactions, mediated by protein tyrosine phosphatases, doesn't just allow feedback control of kinase activity, but can also be involved in sensing stress stimuli (Nguyen and Shiozaki, 1999).

\section{REGULATION OF MAPK ACTIVATION BY THIOREDOXIN}

Although diverse stimuli activate MAPK pathways, in many cases, secondary generation of ROS/redox changes are responsible for increases in MAPK activity. Consequently, amongst the most studied mechanisms are those by which $\mathrm{H}_{2} \mathrm{O}_{2}$ stimulates MAPK acti- vation. The MAPKKK ASK1(MAPKKK5) is important in the activation of both $\mathrm{p3} 8$ and JNK MAPK in response to a variety of stimuli, including $\mathrm{H}_{2} \mathrm{O}_{2}$ which may also act as a second messenger downstream of other stimuli, such as TNF- $\alpha$ (Ichijo et al., 1997, Tobiume et al., 2001). Following identification in a two hybrid screen for ASK1 interactors, the thioredoxin, Trx1, was found to bind to the N-terminus of ASK1, directly inhibiting its activation under reducing conditions (Saitoh et al., 1998). In this study, treatment of cells with TNF- $\alpha$, or for $20 \mathrm{~min}$ with $1 \mathrm{mM}$ $\mathrm{H}_{2} \mathrm{O}_{2}$, was proposed to activate ASK1 by causing Trx1 to become oxidised, promoting its dissociation from ASK1 (Saitoh et al., 1998). ASK1 then oligomerises allowing autophosphorylation of a threonine residue in ASK1's activation loop that increases its kinase activity (Gotoh and Cooper, 1998; Tobiume et al., 2001). Hence this leads to the increased phosphorylation of p38 and JNK MAPK and apoptosis (Ichijo et al., 1997). More recent studies have suggested that ASK1 oligomerisation also involves the $\mathrm{H}_{2} \mathrm{O}_{2}$-induced formation of intermolecular disulphide bonds between cysteines in ASK1 which help stabilise the ASK1-ASK1 interactions required for autophosphorylation (Nadeau et al., 2007). This suggests that the ASK1-inhibitory role of Trx1 may be to reduce these oxidised, oligomeric forms of ASK1, preventing autophosphorylation (Nadeau et al., 2009). 
ASK1 is critically involved in signal transduction to p38 and JNK MAPK in response to a variety of stimuli. In most cases the oxidation state of thioredoxin has not been examined. However, these studies suggest that many activating stimuli may act by causing increased oxidation of thioredoxin. In which case, this would render ASK1 an effective sensor of thioredoxin oxidation. Intriguingly, thioredoxin is actually required for the $\mathrm{H}_{2} \mathrm{O}_{2}$-induced activation of the p38/JNK-related Hog1 MAPK in the fungal pathogen Candida albicans, raising the possibility that, in some cases, oxidised thioredoxin might even play a positive role in MAPK activation (da Silva Dantas et al., 2010).

However, in addition to inhibiting the activity of MAPKKK, thioredoxins are also implicated in feedback mechanisms to reactivate protein tyrosine phosphatases (PTPs) which de-activate MAPK. The catalytic cysteine of PTP becomes oxidised by $\mathrm{H}_{2} \mathrm{O}_{2}$ generated in response to a variety of stimuli. In many cases, irreversible oxidation is prevented by the formation of a disulphide bond with another cysteine which may be reduced by thioredoxin Trx1 (Kwon et al., 2004; Schwertassek et al., 2014). Thioredoxin oxidation would therefore also be predicted to inhibit the reduction of oxidised, inactive PTPs, increasing the extent or duration of MAPK activation.

\section{ROLES OF PRX IN $\mathrm{H}_{2} \mathrm{O}_{2}$ SIGNALLING AND ACTIVATION OF MAPK}

Prx have been shown to have multiple roles in $\mathrm{H}_{2} \mathrm{O}_{2}$-signalling. Prx have been proposed to act as barriers to $\mathrm{H}_{2} \mathrm{O}_{2}$ signal transduction and indeed there is plenty of evidence to suggest that Prx inhibit the activation of ROS-activated signalling pathways, including MAPK (Cao et al., 2009; Choi et al., 2005; Kang et al., 2004; Kil et al., 2012; Woo et al., 2010; Yang et al., 2007). Given that antioxidants, such as $\mathrm{N}$-acetyl cysteine (NAC) and catalase, inhibit MAPK activation, this has led to the conclusion that, in such cases, Prx inhibit activation by removing peroxides (Hashimoto et al., 2001; Yang et al., 2007) (Fig. 2A). Indeed, post-translational mechanisms to inhibit the thioredoxin peroxidase activity of Prx have been shown to be important for signal transduction (Kil et al., 2012; Woo et al., 2010).

Conversely, Prx have also been found to be important for $\mathrm{H}_{2} \mathrm{O}_{2}$ signal transduction. For instance, a genetic screen, using a LacZ reporter under the control of an $\mathrm{H}_{2} \mathrm{O}_{2}$-activated promoter, unexpectedly identified that the peroxiredoxin TSA1 was required for $\mathrm{H}_{2} \mathrm{O}_{2}$-induced gene expression in a commonly used laboratory strain of the budding yeast Saccharomyces cerevisiae (Ross et al., 2000) reflecting TSA1's involvement in the $\mathrm{H}_{2} \mathrm{O}_{2}$-induced activation of the AP1-like transcription factor Yap1 (Okazaki et al., 2005). Subsequent studies have established that the single 2-Cys Prx, Tpx1, is required for $\mathrm{H}_{2} \mathrm{O}_{2}$ signal transduction in the unrelated fission yeast Schizosaccharomyces pombe, suggesting a conserved function for Prx in promoting $\mathrm{H}_{2} \mathrm{O}_{2}$ signal transduction (Veal et al., 2004). Indeed, the thioredoxin peroxidase activity of Tpx1 is required for the $\mathrm{H}_{2} \mathrm{O}_{2}$ induced activation of the Pap1 transcription factor (Bozonet et al., 2005; Vivancos et al., 2005), that mediates the transcriptional response to low levels of $\mathrm{H}_{2} \mathrm{O}_{2}$. Moreover, Tpx1 is also required for the activation of the p38/JNK-related MAPK Sty1 (Spc1) that phosphorylates the Atf1 transcription factor, promoting the Atf1-dependent expression of an overlapping set of stress-protective genes (Veal et al., 2004). Intriguingly, in contrast to Pap1, the activation of Sty1 does not require the thioredoxin peroxidase activity of Tpx1 (Veal et al., 2004). Overexpression of Tpx1 also stimulates the increased phosphorylation of Sty1. Indeed, overexpression of Tpx1 restores inducible phosphorylation to cells expressing a constitutively partially active form of the MAPKK Wis1, indicating that Tpx1 acts downstream of the MAPKK to promote Sty1 activation (Veal et al., 2004) [For a review see (Veal et al., 2014)]

Although it was possible that positive ROS-signalling roles of Prx were confined to unicellular eukaryotes, there is increasing evidence that Prx promote ROS-signalling in animals too. For instance, Prx2 was recently found to act as an $\mathrm{H}_{2} \mathrm{O}_{2}$ receptor, transmitting oxidative signals to the redox-regulated transcription factor STAT3 (Sobotta et al., 2015) (Fig. 2). Prx have also been shown to promote ROS-induced activation of MAPK in animals. For instance, Prdx1 is required for oxidised Low Density Lipoprotein (oxLDL)-induced activation of p38 in macrophage-derived foam cells (Conway and Kinter, 2006), $\mathrm{H}_{2} \mathrm{O}_{2}-$ induced p38 activation in mammalian cells (Jarvis et al., 2012), and for arsenite-induced activation of the PMK-1 MAPK in the nematode worm Caenorhabditis elegans (Olahova et al., 2008). Prdx1 has also been shown to promote p38 activity in pancreatic duct adenocarcinoma cells (Taniuchi et al., 2015). Although the mechanism/s by which Prx promote p38/JNK MAPK activation are not well-established there are a number of possibilities which are discussed below and illustrated in Fig. 2:

[1] As direct redox-transducers: Prdx1 has been found to form intermolecular disulphide bonds with ASK1 that were proposed to initiate the oxidation of ASK1 oligomers (Jarvis et al., 2012). This suggests that in this case Prdx1 is able to act as an $\mathrm{H}_{2} \mathrm{O}_{2}$ receptor transducing the signal to drive the oxidative activation of ASK1. This is reminiscent of the role of Prx2 in promoting the activation of the STAT3 transcription factor (Fig. 2) (Sobotta et al., 2015). STAT3 is activated by transient formation of disulphide-linked STAT3 oligomers which are subsequently reduced by thioredoxin. The detection of Prx2-STAT3 disulphides suggests that Prx2 participates directly in initiating STAT3 oxidation. Although the abundance and $\mathrm{H}_{2} \mathrm{O}_{2}$-reactivity of Prx has led to suggestions that such mechanisms must be prevalent in the $\mathrm{H}_{2} \mathrm{O}_{2}$-induced oxidation of target signalling proteins (Winterbourn, 2008), it remains to be determined whether such peroxidase-based redox relays are widely conserved $\mathrm{H}_{2} \mathrm{O}_{2}$-sensing mechanisms, or limited to specific situations. Intriguingly, in S. pombe Tpx1 also forms mixed disulphides with the Sty1 MAPK (Veal et al., 2004). Indeed, further cysteines in Sty1 have been identified which form an intracellular disulphide bond which is important for transcriptional responses to $\mathrm{H}_{2} \mathrm{O}_{2}$ (Day and Veal, 2010). However, it remains to be determined how Tpx1-Sty1 disulphide formation leads to increased phosphorylation of Sty1.

[2] As $\mathrm{H}_{2} \mathrm{O}_{2}$-dependent thioredoxin inhibitors: Given that thioredoxin is directly responsible for the reduction of Prx disulphides, it is impossible to separate the role of Prx in signalling from that of thioredoxin. For example, in $S$. pombe exposed to $\mathrm{H}_{2} \mathrm{O}_{2}$, Tpx1 disulphides become the main substrate for Trx1. Hence, as thioredoxin reductase (Trr1) levels are limiting, Trx1 becomes completely oxidised and the reduction of Tpx1 and other Trx1 substrates becomes inhibited (Brown et al., 2013, Day et al., 2012). It is yet to be determined whether this ability of Prx to inhibit the oxidoreductase activity of thioredoxin family proteins in response to $\mathrm{H}_{2} \mathrm{O}_{2}$ is generally an important function of Prx. However, the inhibition of the thioredoxin-like protein, Txl1, by $\mathrm{H}_{2} \mathrm{O}_{2}$-induced Tpx1 disulphides, underlays the role of the thioredoxin peroxidase activity of Tpx1 in the $\mathrm{H}_{2} \mathrm{O}_{2}$-induced activation of the AP-1-like transcription factor Pap1 and adaptation to growth under oxidative stress conditions (Brown et al., 2013). Given the important role of Trx1 in reduction of active, oxidised ASK1 oligomers and inactive, oxidised PTPs it is tempting to speculate that Prx might promote MAPK activation in yeast and animals by inhibiting the activity of thioredoxin 
$\boldsymbol{A}$

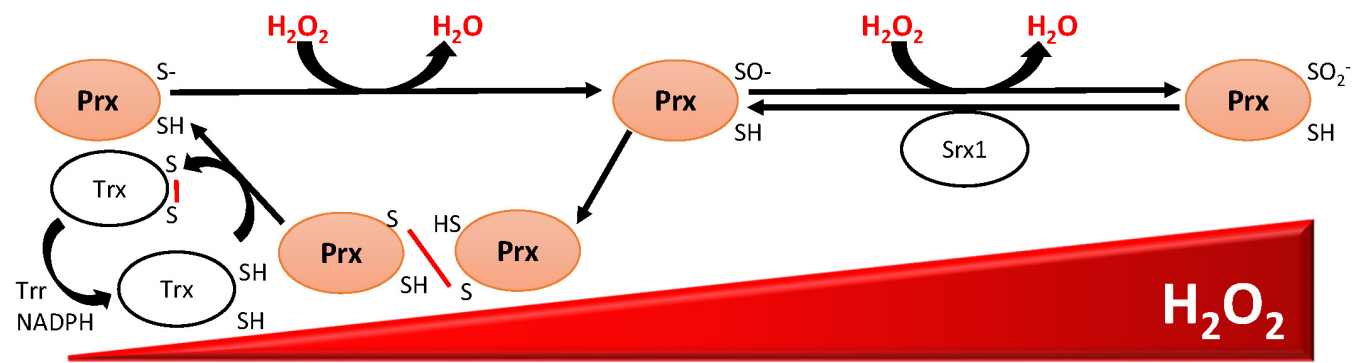

B

[1]

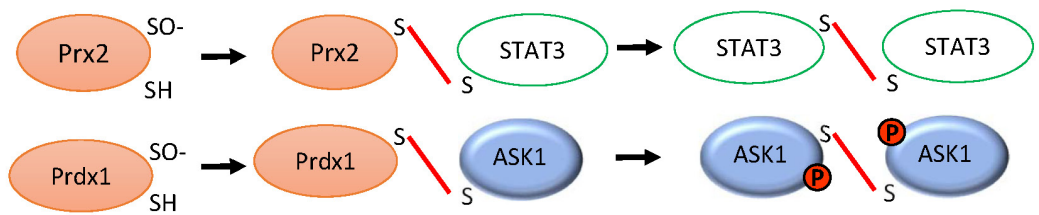

ACTIVE

[2]

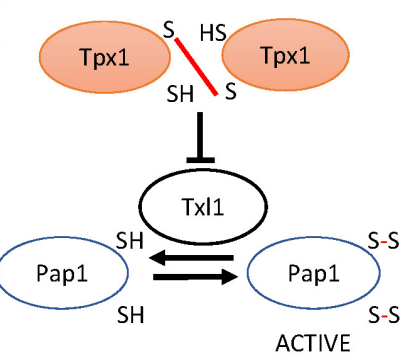

[3]
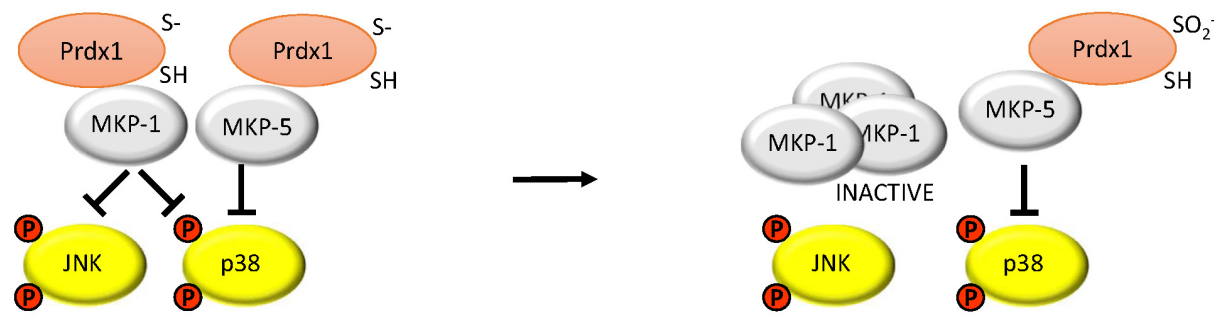

Fig. 2. Mechanisms underlying roles of peroxiredoxin and thioredoxin family proteins in responses to increasing concentrations of hydrogen peroxide, including MAPK activation. (A) Prx can inhibit $\mathrm{H}_{2} \mathrm{O}_{2}$ signalling, including activation of p38 MAPK, by reducing the levels of $\mathrm{H}_{2} \mathrm{O}_{2}$ available to activate these pathways: The peroxidatic cysteine residue of a peroxiredoxin ( $\mathrm{Prx}$ ) reacts with $\mathrm{H}_{2} \mathrm{O}_{2}$ to form a sulphenic acid derivative $\left(-\mathrm{SO}^{-}\right)$. In the catalytic cycle this is followed by the formation of a disulphide bond between this cysteine and the resolving cysteine of a neighbouring Prx. The resulting Prx disulphides are reduced by thioredoxin family proteins ( $\mathrm{Trx}$ ) by thioredoxin reductase (Trr) using electrons from $\mathrm{NADPH}$. At high concentrations of $\mathrm{H}_{2} \mathrm{O}_{2}$, the peroxidatic cysteine can become hyperoxidised to a sulphinic acid (-SO $\mathrm{SO}_{2}^{-}$), which can be reduced by sulphiredoxin ( $\mathrm{Srx} 1$ ) back to the $-\mathrm{SO}^{-}$form. (B) Three mechanisms by which different redox forms of Prx have been shown to promote $\mathrm{H}_{2} \mathrm{O}_{2}$ signalling: [1] Prx2 is able to promote activation of STAT3 through transient formation of Prx2-STAT3 disulphides, which is followed by STAT3STAT3 disulphide-linked oligomers, resulting in STAT3 activation (Sobotta et al., 2015). Similarly, Prdx1 forms disulphide bonds with ASK1, resulting in ASK1-ASK1 oligomers and ASK1 activation (Jarvis et al., 2012). [2] In S. pombe, Tpx1-Tpx1 disulphides are the main substrate for $\operatorname{Trx} 1$, therefore the presence of Tpx1 disulphides prevents the thioredoxin activity of Trx 1 and Txl1 towards other substrates, such as the transcription factor Pap1 (Brown et al., 2013). [3] At low concentrations of $\mathrm{H}_{2} \mathrm{O}_{2}$ in human malignant breast epithelial cells, Prdx1 associates with two MAPK phosphatases, MKP-1 and MKP-5, inhibiting the activity of the MAPK p38, with MKP-1 also dephosphorylating JNK. However, at concentrations of $\mathrm{H}_{2} \mathrm{O}_{2}$ when $\mathrm{Prdx} 1$ is hyperoxidised, $\mathrm{Prdx} 1$ dissociates from MKP-1, resulting in oligomerisation and inactivation of MKP-1 towards p38 and JNK. The association between Prdx1 and MKP-5 is maintained even when Prdx1 is hyperoxidised, allowing MKP-5 to dephosphorylate p38 (Turner-Ivey et al., 2013).

towards these other substrates.

[3] Signalling activity/activities of hyperoxidised Prx: Following exposure of cells to high concentrations of $\mathrm{H}_{2} \mathrm{O}_{2}$, eukaryotic 2-Cys Prx are readily hyperoxidised to a sulphinic acid derivative that cannot be reduced by thioredoxin (Yang et al., 2002). This hyperoxidation is proposed to promote an alternative chaperone activity for Prx (Jang et al., 2004). Hyperoxidation of
Tpx1 in S. pombe allows thioredoxins to reduce other oxidised proteins, and promote cell survival (Day et al., 2012). However, it also means that there is more reduced Txl1 available to reduce Pap1, inhibiting $\mathrm{H}_{2} \mathrm{O}_{2}$-induced Pap1 activation. Nevertheless, Sty1 MAPK becomes increasingly activated, in a Tpx1-dependent manner, as $\mathrm{H}_{2} \mathrm{O}_{2}$ concentrations increase suggesting that hyperoxidised Tpx1 is able to promote Sty1 
activation (Veal et al., 2004). Intriguingly, in human malignant breast epithelial cells, hyperoxidation of $\operatorname{Prdx} 1$ has been shown to differentially affect the activity of two MAP kinase phosphatases (MKP), MKP-1 and MKP-5, that both dephosphorylate $p 38 \alpha$ MAPK, but which favour different substrates with MKP-1 also dephosphorylating JNK kinase (Turner-lvey et al., 2013). Following exposure to high concentrations of $\mathrm{H}_{2} \mathrm{O}_{2}$, the peroxidatic cysteine of $\operatorname{Prdx} 1$ became hyperoxidised, causing it to dissociate from MKP-1. This results in the oligomerisation and inactivation of MKP-1, inhibiting the dephosphorylation of both $\mathrm{p} 38 \alpha$ and JNK MAPKs. In contrast, the Prdx1:MKP-5 complex was maintained, even when Prdx1 was hyperoxidised, protecting MKP-5 from oligomerisation and maintaining its activity towards $\mathrm{p} 38 \alpha$ MAPK. Accordingly, hyperoxidation of $\operatorname{Prdx} 1$ provides a mechanism for specifically increasing JNK activity more than p38 $\alpha$ MAPK activity at high levels of ROS. Intriguingly, the ability of Prdx1 to protect MKP1 and MKP-5 from inactivation is consistent with a potential chaperone function for Prdx1. Alternatively, parallels with our studies in yeast suggest that the hyperoxidation of Prdx1 may also increase the thioredoxin available to maintain MKP-5 in an active reduced state.

\section{CONCLUSION}

Peroxiredoxins and thioredoxin have multiple redox-signalling activities. Here we have attempted to rationalise the basis for both positive and negative effects of Prx on the activation of MAPK pathways. Some outstanding questions remain, for instance: Is the only role of Prx in promoting ASK1 activity due to a direct 'redox-transducing' role or does Prx also promote ASK1 activation by inhibiting thioredoxin from reducing ASK1? Do Prx participate directly in the oxidative inactivation of PTPS either as redox transducers or by promoting the oxidation of thioredoxin? Why, given the high degree of homology between Prdx1 and Prdx2, do they have apparently different effects on MAPK activation? In addition to maintaining MKP-5 activity, do hyperoxidised Prx have other positive signalling/chaperone activities that promote MAPK activation?

Although inhibiting Prx appears to have different effects on MAPK activation depending on the cell or stimuli, in some cases the use of different stimuli, or time-points at which MAPK activation is assessed, make comparisons between studies difficult. In any case, the consequence of inhibiting Prx in any given cell is likely to be determined by the levels of intracellular ROS and intrinsic features of the cell/compartment, such as the capacity to regenerate reduced Prx, using sulfiredoxin, or thioredoxin, using thioredoxin reductase and NADPH (Fig. 2A). For example, it was recently shown that the regulation of mitochondrial sulfiredoxin levels plays an important role in regulating mitochondrial $\mathrm{Prx}$ activity and consequently, cytosolic $\mathrm{H}_{2} \mathrm{O}_{2}$ levels and p38 activity (Kil et al., 2012; 2015). As several studies have illustrated, relative levels and localisation of Prx, thioredoxin and signalling pathway components will also play an important part (Taniuchi et al., 2015; Woo et al., 2010). Accordingly modelling approaches that take into account all these things, developed using quantitative data obtained in wellcharacterised systems, such as yeast, are likely to be extremely important for identifying key features regulating MAPK activity and predicting the response of these signalling pathways to changing redox conditions.

\section{ACKNOWLEDGMENTS}

We are grateful to Brian Morgan for comments on this manuscript and the BBSRC for funding.

\section{REFERENCES}

Bokov, A., Chaudhuri, A., and Richardson, A. (2004). The role of oxidative damage and stress in aging. Mech. Ageing Dev. 125, 811-826.

Bozonet, S.M., Findlay, V.J., Day, A.M., Cameron, J., Veal, E.A., and Morgan, B.A. (2005). Oxidation of a eukaryotic 2-Cys peroxiredoxin is a molecular switch controlling the transcriptional response to increasing levels of hydrogen peroxide. J. Biol. Chem. 280, 23319-23327.

Brown, J.D., Day, A.M., Taylor. S.R., Tomalin, L.E., Morgan, B.A., and Veal, E.A.. (2013). A peroxiredoxin promotes $\mathrm{H} 2 \mathrm{O} 2$ signaling and oxidative stress resistance by oxidizing a thioredoxin family protein. Cell Rep. 5, 1425-1435.

Cao, J., Schulte, J., Knight, A., Leslie, N.R., Zagozdzon, A., Bronson, R., Manevich, Y., Beeson, C., and Neumann, C.A. (2009). Prdx1 inhibits tumorigenesis via regulating PTEN/AKT activity. EMBO J. 28, 1505-1517.

Choi, M.H., Lee, I.K., Kim, G.W., Kim, B.U., Han, Y.H., Yu, D.Y., Park, H.S., Kim, K.Y., Lee, J.S., Choi. C., et al. (2005). Regulation of PDGF signalling and vascular remodelling by peroxiredoxin II. Nature 435, 347-353

Conway, J.P., and Kinter, M. (2006). Dual role of peroxiredoxin I in macrophage-derived foam cells. J. Biol. Chem. 281, 2799128001.

da Silva Dantas, A., Patterson, M.J., Smith, D.A., Maccallum, D.M. Erwig, L.P., Morgan, B.A., and Quinn, J. (2010). Thioredoxin regulates multiple hydrogen peroxide-induced signaling pathways in Candida albicans. Mol. Cell. Biol. 30, 4550-4563.

Day, A.M., and Veal, E.A. (2010). Hydrogen peroxide-sensitive cysteines in the Sty1 MAPK regulate the transcriptional response to oxidative stress. J. Biol. Chem. 285, 7505-7516.

Day, A.M., Brown, J.D., Taylor, S.R., Rand, J.D., Morgan, B.A., and Veal EA. (2012). Inactivation of a peroxiredoxin by hydrogen peroxide is critical for thioredoxin-mediated repair of oxidized proteins and cell survival. Mol. Cell 45, 398-408.

Gotoh, Y., and Cooper, J.A. (1998). Reactive oxygen species- and dimerization-induced activation of apoptosis signal-regulating kinase 1 in tumor necrosis factor-alpha signal transduction. J. Biol. Chem. 273, 17477-17482.

Gutteridge, J.M.C., and Halliwell, B. (1999). Free Radicals in Biology and Medicine. (Oxford, UK: Oxford University Press).

Hashimoto, S., Gon, Y., Matsumoto, K., Takeshita, I., and Horie, T. (2001). N-acetylcysteine attenuates TNF-alpha-induced p38 MAP kinase activation and p38 MAP kinase-mediated IL-8 production by human pulmonary vascular endothelial cells. $\mathrm{Br} \mathrm{J}$. Pharmacol. 132, 270-276.

Holmgren, A., and Lu, J. (2010). Thioredoxin and thioredoxin reductase: current research with special reference to human disease. Biochem. Biophys. Res. Commun. 396, 120-124.

Holmström, K.M., and Finkel, T. (2014). Cellular mechanisms and physiological consequences of redox-dependent signalling. Nat Rev. Mol. Cell Biol. 15, 411-421.

Ichijo, H., Nishida, E., Irie, K., ten Dijke, P., Saitoh, M., Moriguchi, T., Takagi, M., Matsumoto, K., Miyazono, K., and Gotoh, Y. (1997) Induction of apoptosis by ASK1, a mammalian MAPKKK that activates SAPK/JNK and p38 signaling pathways. Science 275 , 90-94.

Jang, H.H., Lee, K.O., Chi, Y.H., Jung, B.G., Park, S.K., Park, J.H., Lee, J.R., Lee, S.S., Moon, J.C., Yun, J.W., et al. (2004). Two enzymes in one; two yeast peroxiredoxins display oxidative stress-dependent switching from a peroxidase to a molecular chaperone function. Cell 117, 625-635.

Jarvis, R.M., Hughes, S.M., and Ledgerwood, E.C. (2012). Peroxiredoxin 1 functions as a signal peroxidase to receive, transduce, and transmit peroxide signals in mammalian cells. Free Radic. Biol. Med. 53, 1522-1530.

Kang, S.W., Chang, T.S., Lee, T.H., Kim, E.S., Yu, D.Y., and Rhee, S.G. (2004). Cytosolic peroxiredoxin attenuates the activation of Jnk and p38 but potentiates that of Erk in Hela cells stimulated with tumor necrosis factor-alpha. J. Biol. Chem. 279, 2535-2543.

Kil, I.S., Lee, S.K., Ryu, K.W., Woo, H.A., Hu, M.C., Bae, S.H., and Rhee, S.G. (2012). Feedback control of adrenal steroidogenesis via $\mathrm{H} 2 \mathrm{O} 2$-dependent, reversible inactivation of peroxiredoxin III in mitochondria. Mol. Cell 46, 584-594.

Kil, I.S., Ryu, K.W., Lee, S.K., Kim, J.Y., Chu, S.Y., Kim, J.H., Park, 
S. and Rhee, S.G. (2015). Circadian oscillation of sulfiredoxin in the mitochondria. Mol. Cell 59, 651-663.

Kwon, J., Lee, S.R., Yang, K.S., Ahn, Y., Kim, Y.J., Stadtman, E.R. and Rhee, S.G. (2004). Reversible oxidation and inactivation of the tumor suppressor PTEN in cells stimulated with peptide growth factors. Proc. Natl. Acad. Sci. USA 101, 16419-16424.

Marshall, C.J. (1994). MAP kinase kinase kinase, MAP kinase kinase and MAP kinase. Curr. Opin. Genet. Dev. 4, 82-89.

Nadeau, P.J., Charette, S.J., Toledano, M.B., and Landry, J. (2007). Disulfide Bond-mediated multimerization of Ask1 and its reduction by thioredoxin-1 regulate $\mathrm{H}(2) \mathrm{O}(2)$-induced c-Jun $\mathrm{NH}(2)$ terminal kinase activation and apoptosis. Mol. Biol. Cell 18, 3903-3913.

Nadeau, P.J., Charette, S.J., and Landry, J. (2009). REDOX reaction at ASK1-Cys250 is essential for activation of JNK and induction of apoptosis. Mol. Biol. Cell 20, 3628-3637.

Nguyen, A.N., and Shiozaki, K. (1999). Heat-shock-induced activation of stress MAP kinase is regulated by threonine- and tyrosine-specific phosphatases. Genes Dev. 13, 1653-1663.

Okazaki, S., Naganuma, A., and Kuge, S. (2005). Peroxiredoxinmediated redox regulation of the nuclear localization of Yap1, a transcription factor in budding yeast. Antioxid. Redox Signal. 7 , 327-334.

Oláhová, M., Taylor, S.R., Khazaipoul, S., Wang, J., Morgan, B.A., Matsumoto, K., Blackwell, T.K., and Veal, E.A. (2008). A redoxsensitive peroxiredoxin that is important for longevity has tissueand stress-specific roles in stress resistance. Proc. Natl. Acad. Sci. USA 105, 19839-19844

Ross, S.J., Findlay, V.J., Malakasi, P., and Morgan, B.A. (2000). Thioredoxin peroxidase is required for the transcriptional response to oxidative stress in budding yeast. Mol. Biol. Cell 11, 2631-2642.

Sabio, G., and Davis, R.J. (2014). TNF and MAP kinase signalling pathways. Semin. Immunol. 26, 237-245.

Saitoh, M., Nishitoh, H., Fujii, M., Takeda, K., Tobiume, K., Sawada, Y., Kawabata, M., Miyazono, K., and Ichijo, H. (1998). Mammalian thioredoxin is a direct inhibitor of apoptosis signal-regulating kinase (ASK) 1. EMBO J. 17, 2596-2606.

Schwertassek, U., Haque, A., Krishnan, N., Greiner, R., Weingarten, L., Dick, T.P., and Tonks, N.K. (2014). Reactivation of oxidized PTP1B and PTEN by thioredoxin 1. FEBS J, 281, 3545-3558.

Sobotta, M.C., Liou, W., Stöcker, S., Talwar, D., Oehler, M., Ruppert, T., Scharf, A.N., and Dick, T.P. (2015). Peroxiredoxin-2 and STAT3 form a redox relay for $\mathrm{H} 2 \mathrm{O} 2$ signaling. Nat. Chem. Biol. 11, 64-70.

Taniuchi, K., Furihata, M., Hanazaki, K., Iwasaki, S., Tanaka, K.,
Shimizu, T. Saito, M., and Saibara, T. (2015). Peroxiredoxin 1 promotes pancreatic cancer cell invasion by modulating p38 MAPK activity. Pancreas 44, 331-340.

Tobiume, K., Matsuzawa, A., Takahashi, T., Nishitoh, H., Morita, K., Takeda, K., Minowa, O., Miyazono, K., Noda, T., and Ichijo, H. (2001). ASK1 is required for sustained activations of JNK/p38 MAP kinases and apoptosis. EMBO Rep. 2, 222-228.

Toone, W.M., and Jones, N. (1998). Stress-activated signalling pathways in yeast. Genes Cells 3, 485-498.

Turner-Ivey, B., Manevich, Y., Schulte, J., Kistner-Griffin, E., Jezierska-Drutel, A., Liu, Y., and Neumann, C.A. (2013). Role for Prdx1 as a specific sensor in redox-regulated senescence in breast cancer. Oncogene 32, 5302-5314

Veal, E., and Day, A. (2011). Hydrogen peroxide as a signaling molecule. Antioxid. Redox Signal. 15, 147-151.

Veal, E.A., Findlay, V.J., Day, A.M., Bozonet, S.M., Evans, J.M., Quinn, J., and Morgan, B.A. (2004). A 2-Cys peroxiredoxin regulates peroxide-induced oxidation and activation of a stressactivated MAP kinase. Mol. Cell 15, 129-139.

Veal, E.A., Tomalin, L.E., Morgan, B.A., and Day, A.M. (2014). The fission yeast Schizosaccharomyces pombe as a model to understand how peroxiredoxins influence cell responses to hydrogen peroxide. Biochem. Soc. Trans. 42, 909-916.

Vivancos AP1, Castillo EA, Biteau B, Nicot C, Ayté J, Toledano MB Hidalgo E. (2005). A cysteine-sulfinic acid in peroxiredoxin regulates $\mathrm{H} 2 \mathrm{O} 2$-sensing by the antioxidant Pap1 pathway. Proc. Natl. Acad. Sci. USA 102, 8875-8880.

Winterbourn, C.C. (2008). Reconciling the chemistry and biology of reactive oxygen species. Nat. Chem. Biol. 4, 278-286.

Woo, H.A., Yim, S.H., Shin, D.H., Kang, D., Yu, D.Y., and Rhee, S.G (2010). Inactivation of peroxiredoxin I by phosphorylation allows localized $\mathrm{H}(2) \mathrm{O}(2)$ accumulation for cell signaling. Cell 140, 517528.

Wood, Z.A., Schröder, E., Robin Harris, J., and Poole, L.B. (2003). Structure, mechanism and regulation of peroxiredoxins. Trends Biochem. Sci. 28, 32-40.

Yang, C.S., Lee, D.S., Song, C.H., An, S.J., Li, S., Kim, J.M., Kim, C.S., Yoo, D.G., Jeon, B.H., Yang, H.Y., et al. (2007). Roles of peroxiredoxin II in the regulation of proinflammatory responses to LPS and protection against endotoxin-induced lethal shock. J. Exp. Med. 204, 583-594

Yang, K.S., Kang, S.W., Woo, H.A., Hwang, S.C., Chae, H.Z., Kim, K., and Rhee, S.G. (2002). Inactivation of human peroxiredoxin during catalysis as the result of the oxidation of the catalytic site cysteine to cysteine-sulfinic acid. J. Biol. Chem. 277, 3802938036 